\title{
The influence of social capital on self-rated health and depression - The Nord-Trøndelag health study (HUNT)
}

\author{
Erik R. Sund, ${ }^{1}$ Stig H. Jørgensen, ${ }^{1}$ Andy Jones, ${ }^{2}$ Steinar Krokstad ${ }^{3}$ and Marit Heggdal ${ }^{4}$ \\ ${ }^{1}$ Department of Geography, Norwegian University of Science and Technology (NTNU), NO-7491 Trondheim, Norway \\ ${ }^{2}$ School of Environmental Sciences, University of East Anglia, Norwich, Norfolk, NR4 7TJ, UK \\ ${ }^{3}$ Department of Community Medicine and General Practice, Norwegian University of Science and Technology \\ (NTNU), HUNT Research Centre, Verdal, Norway \\ ${ }^{4}$ Faculty of Medicine, Norwegian University of Science and Technology (NTNU), NO-7491 Trondheim, Norway
}

Correspondence: Erik R. Sund, E-mail: erik.r.sund@svt.ntnu.no

\begin{abstract}
The article examines the relationship between neighbourhood social capital and two health outcomes: selfrated health and depression. A total of 42,571 individuals aged 30-67 years participated in a cross-sectional total population health study in Nord-Trøndelag in 1995-1997 (HUNT II) and were investigated using multilevel modelling. Aims were, first, to investigate potential area effects after accounting for the characteristics of individuals in the neighbourhoods $(\mathrm{N}=155)$, and, second, to explore the relationships between contextual social capital (the level of trust at the neighbourhood level and the level of local organizational activity) and the two health measures. Models with stepwise inclusion of individual level factors attenuated the ward level variance for both self-rated health (PCV: 41\%) and depression (PCV: 43\%). The inclusion of the two contextual social capital items attenuated the ward level variance for both self-rated health and depression, however to varying degrees. At the individual level, contextual social capital was associated with both self-rated health and depression. Individuals living in wards with a low level of trust experienced an increased risk of 1.36 (95\% CI: 1.13-1.63) for poor self-rated health compared to individuals in wards with a high level of trust. For depression, this effect was even stronger (OR 1.52, 1.23-1.87). The associations with the level of organizational activity were inconsistent and weaker for both health outcomes. It was concluded that geographical variations in self-rated health and depression are largely due to the socioeconomic characteristics of individuals. Nevertheless, contextual social capital, expressed as the level of trust, was found to be associated with depression and self-rated health at individual level.
\end{abstract}

\section{INTRODUCTION}

The proposition that 'where you live' matters for health and longevity has been advanced by epidemiologists, demographers and geographers (1). Geographical variations in health were initially attributed to differences in the socio-demographic characteristics of individuals. However, features of the areas themselves can also contribute, independently of the individuals involved, and these are referred to as contextual explanations or contextual effects $(2,3)$. Also, observations of social class gradients in health and mortality in Western societies have received increasing attention. Regardless of type of indicator used to operationalize socio-economic position (SEP), the inverse and graded association between SEP and health have been found in virtually all populations (4). Both in the area-effects debate and the related health-inequality debate, the concept of social capital has re-emerged as an essential concept. It is typically regarded as a part of societal structure and a key determinant of the health of populations (5). In an attempt to combine both spatial and social elements, social capital might be the key concept to explain both geographical inequalities and health inequalities between social positions in society (6).

The main aims of this article are twofold. The first aim is to investigate whether geographical differences in health are a compositional artefact or due to contextual influences. Are geographical differences merely spatial manifestations of social inequalities in health? The second aim is to test the social capital and health relationship with two different health measures at a very low geographical level. The ward level used in these analyses is possibly in good correspondence with an individual's perception of what constitutes their neighbourhood. The underlying idea for this approach is that place makes a separate and distinct contribution to both spatial and social health inequalities.

\section{SOCIAL CAPITAL, HEALTH AND AREA EFFECTS}

The idea that group level factors are important to the health of individuals is not new (7-9). In his seminal paper 'Sick individuals and sick populations', Rose (10) discusses the importance of detecting group level 
factors (or population-level factors as he terms them). By focusing on what distinguishes sick from healthy individuals within a population, studies may miss important health determinants, but by comparing different populations these factors may be detected. Hence Rose suggested that the causes of disease in individuals may be distinguished from the causes of differences in disease rates between populations (groups), and this may be of great importance for disease prevention and particularly for understanding health inequalities (11). As Schwartz and Carpenter explained: 'When the causes of the rate differences between populations and the causes of interindividual variation within a population are different and the question is about rate differences, this discrepancy results in what has been called a type III error - providing a right answer for the wrong question' (12, p. 1175).

The resurgence of interest in the social determinants of health has contributed to highlighting the importance of group level factors, with Wilkinson as an exponent in revitalizing this perspective (13-15). Summarizing some complex debates, he has argued that beyond a certain level of development, the most egalitarian societies have the best health standing. Wilkinson contends that egalitarian societies possess a higher level of social capital - simply stated, they are more cohesive. The social capital concept differs among theorists. Broadly speaking, it relates to social interaction and network membership, and their consequences (16). Wilkinson further argues that living in an equal society reduces the level of psychosocial stress resulting from pronounced social hierarchies. Partly as a result of Wilkinson's work, a separate body of research has emerged which is less focused on income inequalities and more oriented towards social capital, the 'standalone' social-capital-and-health hypothesis.

Social capital is a multidimensional concept, and following Putnam's definition it consists of 'features of interpersonal trust, norms of reciprocity, and social engagement that foster community and social participation' (17). The concept is usually further refined into structural (e.g. participation) and cognitive (e.g. trust) components, i.e. different forms of social capital. These can be described as 'what individuals do' as opposed to 'what individuals feel' (18). The beneficial effects to individuals include the provision of affective support and a source of self-esteem and mutual respect. Further, social capital can facilitate access to services and amenities, promote healthy behaviour, exhibit social control over deviant health-related behaviour, and ease the transmission of health information (19).

According to Wilkinson's view on social capital as an outcome of hierarchical societies, there are also direct physiological effects (embodiment) on individuals' psychoneuroendocrine systems through stress, physical hardship and emotional difficulties (13). This has led Wilkinson and others to suggest that psychosocial mechanisms are the key element $(20,21)$, and one type of critique of proposed mechanisms has been expressed from the 'neo-materialists' $(22,23)$. They claim the social capital (and psychosocial) literature obscures underlying political, administrative and economic determinants. The critique is directed against Wilkinson's income-inequality-and-social-capital hypothesis as well as the stand-alone social capital literature. However, the criticism is much sharper towards the latter because it allegedly omits structural economic inequality and political conflict (24). Nevertheless, as exponents for both sides in this vigorous debate acknowledge, separating the material from the psychosocial is not sustainable $(24,25)$. Both sides in the debate are rooted in the common perception that inequality is divisive and socially corrosive. It should also be noted that there are differences between Putnam's and Wilkinson's notion of social capital, especially regarding what kind of processes influence social capital formation and decline.

Studies of the importance of the social environment have shown that living in an area with a high level of social capital or related concepts is associated with a number of social phenomena: the quality of democracy (26), voting patterns (27), well-being (28), and crime $(29,30)$. Health associations have been demonstrated with regard to mortality $(31,32)$, coronary heart disease (33), obesity and physical inactivity (34), and a number of other health outcomes. Of particular relevance for the current study are two reviews of the social capital and mental health relationship $(35,36)$. While Whitley and McKenzie conclude on inadequate evidence supporting social capital's influence on individuals' mental health, Almedom finds that social capital is salutary for adults' mental health. As regards self-rated health, studies are both supporting (37-39) and dismissing an association $(40,41)$. The variety of definitions, conceptualizations, operationalizations, and health outcome under study can possibly explain this discrepancy. Regarding the latter, there are a number of justifications for the current study's choice of health outcomes.

Depression is a highly prevalent disorder in the general population, and constitutes a major public health problem (42). The economic burden for society at large imposed by mental health problems is huge, both in terms of disability expenditure and loss of productivity. Approximately one-third of disability pensions awarded in Norway are based on mental health problems and are increasing both in absolute and relative terms (43). Depression has been found to be a robust predictor for disability pension awards (44).

Self-rated health is one of the most common health measures in health research. It is considered a valid and reliable indicator and a very cost-effective means of individual health assessment that provides valuable additional information to other health measures (45). An individual's assessment of their own health thus represents a summary statement of perceived aspects of subjective as well as objective health. Self ratings of health have also proved to be an important predictor 
for survival (46) and use of health care (47). A study utilizing the same data material as the current study demonstrated the predictive power of self ratings for subsequent mortality (48).

\section{Methods}

\section{Data material}

In the Norwegian county of Nord-Trøndelag, two large-scale, total population (20+ years of age) health surveys have been conducted (HUNT). The present study analyses participants from the second survey in 1995-97 (HUNT II) and includes individuals of 30-67 years of age, comprising a total of 42,571 males and females. The individuals were nested within 155 wards.

\section{Definitions: individual level}

\section{Outcomes:}

Self-rated health was dichotomized into poor health (poor and fair) and good health (very good and good) based on the first questionnaire item: How is your present state of health?

Depression was derived from the Hospital Anxiety and Depression Scale (HADS). This is a self-report questionnaire consisting of seven four-point Likert-scaled items for depression. The HADS-instrument has shown good case-finding properties (49). A cut-off value of 8 on the depression subscale has been found to give an optimal balance between sensitivity and specificity and was therefore utilized in the present study.

\section{Independent variables:}

Age was classified into the four age categories: $30-39$, 40-49, 50-59, and 60-67.

Sex: female and male.

Civil status was dichotomized as: (1) married (plus registered partner) versus (2) not married (plus widowed, divorced and separated).

Education comprised three groups: (1) Low: 7-9 years (no high school) and 10-11 years (some high school), (2) Medium: 12 years (completed high school), and (3) High: $>12$ years (College/University).

Employed was derived from the question: What kind of work situation do you have? This variable was dichotomized into (1) Employed (paid work and selfemployed in business) and (2) Not employed (full-time housework, education/military service, unemployed/ certified unfit for work, and retired/receiving social security). The respondents could fill in one or more responses to this question - hence all respondents who put a mark against paid work and/or self-employed in business were classified as employed.

\section{Definitions: neighbourhood level}

Social trust is based on the questionnaire statement: 'One cannot rely on each other here' (completely agree, partially agree, not sure, partially disagree, completely disagree). These were dichotomized into: (1)
Trust (partially/completely disagree) and (2) No trust (completely/partially agree and not sure). The respondents were requested to relate their answer according to their neighbourhood/area of residence. The proportion reporting trust in each ward represents the level of neighbourhood trust. The wards were further classified into four categories based on the mean and \pm 1 SD. Wards with less than 30 individuals were excluded from the analysis.

Participation is derived from the question: 'How often do you take part in social activities such as, for example, sewing circle, athletic club, political association, religious or other associations?' The responses were dichotomized into: (1) Participation (1-2 times a month, about once a week, more than once a week) and (2) No participation (never/a few times a year). The proportion of individuals reporting participation in each ward represents participation in the respective neighbourhood/ward. The variable was classified similar to the social trust variable, into four categories (based on mean and $\pm 1 \mathrm{SD}$ ). As with the trust variable, we excluded wards with less than 30 individuals from the analysis.

\section{Statistics}

Multilevel logistic regression models (two-level variance components models with random intercepts) with individuals (first level) nested within wards (second level) were fitted to the two outcomes in separate analyses. The baseline (null-model) contained no predictor variables. In the next model, individual level covariates were included, and in the last the two models, ecological social capital variables were fitted separately. The models were estimated using MLwiN software (Version 2.02 (50)), based on the penalized quasi-likelihood approximation (PQL) of a second-order Taylor linearization procedure. The binomial logit function was used in the models with the dichotomous outcome being set to record whether or not each respondent reported poor self-rated health or depression and an odds ratio of reporting these outcomes being estimated for each of the explanatory variables. The odds ratios hence constitute the models' fixed effects.

Random effects is reported as between ward variance (with standard errors). In addition, the Variance Partition Coefficient (VPC) is calculated. The VPC is the proportion of the total variance associated with the wards (second level). The VPC is calculated with the latent variable method (51) as:

$$
\mathrm{VPC}=\mathrm{V}_{\mathrm{N}} / \mathrm{V}_{\mathrm{N}}+\mathrm{V}_{\mathrm{I}}\left(\mathrm{V}_{\mathrm{I}}=\pi^{2} / 3\right)
$$

$\mathrm{V}_{\mathrm{N}}$ is the neighbourhood variance (second level variance) and $V_{I}$ is the variance between individuals. The VPC ranges between 0 and 1 and a high value informs that areas are very important whilst a VPC of 0 suggests that the wards (area level) are similar to random samples taken from the county. Put simply, the VPC is a measure of the extent to which people within groups are more alike than across groups. 
Ward differences in health may be attributable to both context (place) and characteristics of individuals. By adjusting for individual characteristics and comparing different models, the Proportional Change in Variance $(\mathrm{PCV})$ quantifies how much of the initial area effect (second level variance) can be explained by the characteristics of individuals. The PCV is calculated for the different models as (52):

$$
\mathrm{PCV}=\left(\mathrm{V}_{0}-\mathrm{V}_{1} / \mathrm{V}_{0}\right) \times 100
$$

The $\mathrm{V}_{0}$ is the neighbourhood variance in the initial null model and $\mathrm{V}_{1}$ is the neighbourhood variance in the consecutive models.

\section{RESULTS}

The descriptives for the individuals and the wards are summarized in Table 1. Missing values on the covariates were negligible, while on symptoms of depression the proportion missing was more pronounced (7\%). The mean population in the wards was 275 (range 312503). The level of trust varied from about $30 \%$ to about $80 \%$ (mean 60 ), and the mean level of participation was $58 \%$ (range $35-77$ ).

Bivariate associations between the health outcomes and the covariates are shown in Table 2. One-quarter $(25.5 \%)$ reported poor self-rated health. On symptoms of depression, the prevalence was $10.5 \%$. For the ecological variables the tendencies are in the expected direction with better health in the wards defined as having much social capital, and then gradually decreasing. The level of trust in the wards shows the expected associations between health outcomes, and for the level of participation the prevalences are as expected, yet somewhat more modest. Most noteworthy are the associations between labour market participation (employed or not employed) and the health outcomes. The age group included in the analysis are all in the working age group and although the non-employees are a rather heterogeneous group (as defined in this analysis), the associations seem quite strong.

Tables 3 and 4 show the individual- and area characteristics' fixed and random effects for self-rated health and depression. The models were built sequentially, starting with a baseline model with no predictor variables to assess whether multilevel models were necessary. The random effects were significant for both self-rated health (Wald test $p<0.05$ ) and depression (Wald test $p<0.05$ ), suggesting that place of residence matters for these health outcomes.

Starting with self-rated poor health in Table 3 , the amount of variance associated with area or place of residence (model 1$)$ was approximately $2 \%(\mathrm{VPC}=1.9$, variance: 0.063 (SE: 0.011)). By adding individual level predictors, we were able to assess the relative importance of these factors and the level 2 variance gradually decreased with adjustment for sociodemographic characteristics. The relative importance regarding area effects of these individual level covariates can be monitored by the proportional change in level 2 variance $(\mathrm{PCV})$. We noted that $41 \%$ of the level 2 variance is accounted for after adjusting for the characteristics of individuals.

For the social capital measures we observed that the level of trust in the wards is associated with poor self-rated health. Individuals living in wards more than one standard deviation below the average on the level of trust scale (i.e. low trust), have an increased risk of approximately 1.36 (95\% CI: 1.13-1.63) compared to individuals in high trust wards. For the other two categories only the medium-low category (wards between the average and one standard deviation below the

Table 1. Descriptives for individual $(\mathrm{N}=42,571)$ and area level variables $(\mathrm{N}=155)$.

\begin{tabular}{|c|c|c|}
\hline Individual level variables & Number & $(\%)$ \\
\hline \multicolumn{3}{|l|}{ Age } \\
\hline $30-39$ & 11571 & $(27.2)$ \\
\hline $40-49$ & 13468 & $(31.6)$ \\
\hline $50-59$ & 10499 & $(24.7)$ \\
\hline $60-67$ & 7033 & $(16.5)$ \\
\hline Total & 42571 & $(100)$ \\
\hline \multicolumn{3}{|l|}{ Sex } \\
\hline Male & 20323 & $(47.7)$ \\
\hline Female & 22248 & $(52.3)$ \\
\hline Total & 42571 & $(100)$ \\
\hline \multicolumn{3}{|l|}{ Civil status } \\
\hline Married/reg.partner & 30506 & $(71.1)$ \\
\hline Not married & 12064 & $(28.3)$ \\
\hline Missing & 1 & $(0.0)$ \\
\hline Total & 42571 & $(100)$ \\
\hline \multicolumn{3}{|l|}{ Education } \\
\hline Basic/secondary & 29446 & $(69.2)$ \\
\hline Junior college & 2990 & $(7.0)$ \\
\hline University & 8963 & $(21.1)$ \\
\hline Missing & 1172 & $(2.8)$ \\
\hline Total & 42571 & $(100)$ \\
\hline \multicolumn{3}{|l|}{ Employed } \\
\hline Yes & 32791 & $(77.0)$ \\
\hline No & 9140 & $(21.5)$ \\
\hline Missing & 640 & $(1.5)$ \\
\hline Total & 42571 & $(100)$ \\
\hline \multicolumn{3}{|l|}{ Self rated health } \\
\hline Good & 31478 & $(73.9)$ \\
\hline Poor & 10755 & $(25.3)$ \\
\hline Missing & 338 & $(0.8)$ \\
\hline Total & 42571 & $(100)$ \\
\hline \multicolumn{3}{|l|}{ Depressive symptoms } \\
\hline No & 35515 & $(83.4)$ \\
\hline Yes & 4155 & $(9.8)$ \\
\hline Missing & 2901 & $(6.8)$ \\
\hline Total & 42571 & $(100)$ \\
\hline Area level variables & Mean (SD) & Range \\
\hline Population & $275(345)$ & $31-2503$ \\
\hline Level of trust & $60(8.9)$ & $29.6-80.0$ \\
\hline Level of organizational activity & $58(8.5)$ & $34.7-77.4$ \\
\hline
\end{tabular}


Table 2. Bivariate associations between self rated poor health, depression and individual and area factors.

\begin{tabular}{|c|c|c|c|c|}
\hline \multirow{2}{*}{$\overline{\text { Age }}$} & \multicolumn{2}{|c|}{$\begin{array}{c}\text { Number (valid \%) } \\
\text { poor self rated health }\end{array}$} & \multicolumn{2}{|c|}{$\begin{array}{c}\text { Number (valid \%) } \\
\text { depressive symptoms }\end{array}$} \\
\hline & & & & \\
\hline $30-39$ & 1622 & $(14.1)$ & 773 & $(7.0)$ \\
\hline $40-49$ & 2794 & (20.9) & 1259 & $(9.9)$ \\
\hline $50-59$ & 3377 & $(32.5)$ & 1262 & $(13.0)$ \\
\hline $60-67$ & 2962 & $(42.5)$ & 861 & $(14.1)$ \\
\hline Total & 10755 & $(25.5)$ & 4155 & $(10.5)$ \\
\hline \multicolumn{5}{|l|}{ Sex } \\
\hline Male & 4687 & $(23.2)$ & 2075 & $(10.9)$ \\
\hline Female & 6068 & $(27.5)$ & 2080 & $(10.1)$ \\
\hline Total & 10755 & $(25.5)$ & 4155 & $(10.5)$ \\
\hline \multicolumn{5}{|l|}{ Civil status } \\
\hline Married & 7688 & $(25.4)$ & 2854 & $(10.0)$ \\
\hline Not married & 3067 & $(25.6)$ & 1301 & $(11.8)$ \\
\hline Total & 10755 & $(25.5)$ & 4155 & $(10.5)$ \\
\hline \multicolumn{5}{|l|}{ Education } \\
\hline University & 1346 & $(15.1)$ & 587 & (6.8) \\
\hline Junior college & 524 & $(17.7)$ & 206 & $(7.2)$ \\
\hline Basic/secondary & 8411 & $(28.8)$ & 3252 & $(11.8)$ \\
\hline Total & 10281 & $(25.0)$ & 4045 & $(10.4)$ \\
\hline \multicolumn{5}{|l|}{ Employed } \\
\hline Yes & 6074 & $(18.7)$ & 2687 & $(8.6)$ \\
\hline No & 4410 & $(48.7)$ & 1380 & $(17.0)$ \\
\hline Total & 10484 & $(25.2)$ & 4067 & $(10.3)$ \\
\hline \multicolumn{5}{|l|}{ Level of trust in wards $(\%)$} \\
\hline High $(>69.1)$ & 702 & $(22.9)$ & 264 & $(9.1)$ \\
\hline Med high (60.2-69) & 6382 & $(24.1)$ & 2423 & $(9.7)$ \\
\hline Med low (51.3-60.1) & 3046 & $(28.3)$ & 1206 & $(12.1)$ \\
\hline Low $(<51.2)$ & 625 & $(32.1)$ & 262 & $(14.5)$ \\
\hline Total & 10755 & $(25.5)$ & 4155 & $(10.5)$ \\
\hline \multicolumn{5}{|c|}{ Level of participation in wards (\%) } \\
\hline High $(>66.2)$ & 741 & $(24.1)$ & 273 & $(9.5)$ \\
\hline Med high (57.6-66.1) & 4269 & $(23.6)$ & 1740 & $(10.2)$ \\
\hline Med low (49.1-57.5) & 4579 & $(26.5)$ & 1696 & $(10.4)$ \\
\hline Low $(<49)$ & 1166 & $(30.4)$ & 446 & $(12.6)$ \\
\hline Total & 10755 & $(25.5)$ & 4155 & $(10.5)$ \\
\hline
\end{tabular}

average) are significantly different from the reference category (OR: 1.24, 1.08-1.44). The association with structural social capital is substantially weaker; only the category termed low organizational activity is significantly associated with poor self-rated health in model 4. We also note that their contributions in explaining level 2 variance are different.

For depression (Table 4) the level 2 variance in the empty model was 0.051 (SE: 0.012 ), giving a variance partition coefficient of 1.5 . This variance gradually diminished in the consecutive models, and the proportional change in level 2 variance gradually increased while adjusting for the characteristics of individuals and features of the area. Adjustment for individual factors explained $43 \%$ of the level 2 variance, and with the inclusion of the trust variable in model $3,71 \%$ of the level 2 variance was accounted for.
Thus, the inclusion of the ecological trust variable shows a similar, yet stronger, association with depression than with self-rated health. For individuals living in low trust wards the relative risk of depression is approximately $50 \%$ higher compared to the reference category, and residing in what we term medium-low areas exerts a $30 \%$ increased risk of depression. In model 4 the inclusion of the organizational activity variable demonstrates incoherent associations; only the category termed low organizational activity was associated with depression (OR: 1.28, 1.04-1.58).

\section{Discussion}

Summarizing the findings along with possible limitations in this study, we found in accordance with our first research question that geographical inequalities in 
Table 3. Multilevel logistic regression of poor self rated health, men and women 30-67 years HUNT II. 42,571 individuals nested within 155 wards. Odds ratios $(95 \% \mathrm{CI})$.

\begin{tabular}{|c|c|c|c|c|c|c|c|c|c|}
\hline & & \multirow{2}{*}{\multicolumn{2}{|c|}{$\begin{array}{c}\text { Model } 1 \\
\text { Empty }\end{array}$}} & \multicolumn{2}{|c|}{ Model 2} & \multicolumn{2}{|c|}{ Model 3} & \multicolumn{2}{|c|}{ Model 4} \\
\hline & & & & OR & $(95 \% \mathrm{CI})$ & OR & $(95 \% \mathrm{CI})$ & OR & $(95 \% \mathrm{CI})$ \\
\hline \multicolumn{10}{|c|}{ Fixed effects } \\
\hline \multicolumn{10}{|c|}{ Individual level } \\
\hline Age & $30-39$ & & & 1 & Ref & 1 & Ref & 1 & Ref \\
\hline & $40-49$ & & & 1.55 & $(1.43-1.67)$ & 1.54 & $(1.43-1.67)$ & 1.55 & $(1.43-1.67)$ \\
\hline & $50-59$ & & & 2.44 & $(2.24-2.65)$ & 2.43 & $(2.24-2.64)$ & 2.44 & $(2.24-2.64)$ \\
\hline & $60-67$ & & & 2.87 & $(2.57-3.20)$ & 2.86 & $(2.57-3.20)$ & 2.87 & $(2.57-3.20)$ \\
\hline \multicolumn{10}{|l|}{ Sex } \\
\hline & Males & & & 1 & Ref & 1 & Ref & 1 & Ref \\
\hline & Females & & & 1.13 & $(1.08-1.19)$ & 1.13 & $(1.08-1.19)$ & 1.13 & $(1.08-1.19)$ \\
\hline \multicolumn{10}{|c|}{ Civil status } \\
\hline & Married & & & 1 & Ref & 1 & Ref & 1 & Ref \\
\hline & Not married & & & 1.10 & $(1.04-1.16)$ & 1.09 & $(1.04-1.16)$ & 1.09 & (1.04-1.16) \\
\hline \multicolumn{10}{|c|}{ Education } \\
\hline & University & & & 1 & Ref & 1 & Ref & 1 & Ref \\
\hline & Junior College & & & 1.26 & $(1.12-1.42)$ & 1.26 & $(1.12-1.42)$ & 1.26 & $(1.12-1.42)$ \\
\hline & Basic,- Second. School & & & 1.56 & $(1.46-1.67)$ & 1.55 & $(1.45-1.66)$ & 1.56 & $(1.46-1.67)$ \\
\hline \multicolumn{10}{|c|}{ Employed } \\
\hline & Yes & & & 1 & Ref & 1 & Ref & 1 & Ref \\
\hline & No & & & 2.60 & $(2.27-2.97)$ & 2.58 & $(2.26-2.96)$ & 2.59 & $(2.26-2.97)$ \\
\hline \multicolumn{10}{|c|}{ Interaction Age x Employed } \\
\hline & Age $30-39 \times$ Not employed & & & 1 & Ref & 1 & Ref & 1 & Ref \\
\hline & Age $40-49 \times$ Not employed & & & 1.74 & $(1.46-2.09)$ & 1.75 & $(1.46-2.09)$ & 1.74 & $(1.46-2.09)$ \\
\hline & Age 50-59 x Not employed & & & 1.60 & $(1.35-1.90)$ & 1.60 & $(1.35-1.90)$ & 1.60 & $(1.35-1.90)$ \\
\hline & Age $60-67 \times$ Not employed & & & 0.88 & $(0.74-1.05)$ & 0.88 & $(0.74-1.05)$ & 0.88 & $(0.74-1.05)$ \\
\hline \multicolumn{10}{|c|}{ Ward level } \\
\hline \multicolumn{10}{|c|}{ Cognitive social capital } \\
\hline & High trust & & & & & 1 & Ref & & \\
\hline & Medium high & & & & & 1.10 & $(0.96-1.26)$ & & \\
\hline & Medium low & & & & & 1.24 & $(1.08-1.44)$ & & \\
\hline & Low trust & & & & & 1.36 & $(1.13-1.63)$ & & \\
\hline \multicolumn{10}{|c|}{ Structural social capital } \\
\hline & High organizational activity & & & & & & & 1 & Ref \\
\hline & Medium high & & & & & & & 0.99 & $(0.86-1.14)$ \\
\hline & Medium low & & & & & & & 1.07 & $(0.92-1.23)$ \\
\hline & Low organizational activity & & & & & & & 1.19 & $(1.00-1.42)$ \\
\hline \multicolumn{10}{|c|}{ Random effects } \\
\hline Level & 2 variance $(\mathrm{SE})$ & 0.063 & $(0.011)$ & 0.037 & $(0.008)$ & 0.030 & $(0.007)$ & 0.033 & $(0.007)$ \\
\hline VPC & & 1.9 & & 1.1 & & 0.9 & & 1.0 & \\
\hline $\mathrm{PCV}$ & & Ref & & $41 \%$ & & $52 \%$ & & $48 \%$ & \\
\hline
\end{tabular}

health were reduced when we adjusted for the characteristics of individuals. More than $40 \%$ of the second level variation is explained by adjusting for the characteristics of the individuals for both health outcomes. Hence we can claim that geographical inequalities in health are partially spatial manifestations of social inequalities in health at the individual level. Nevertheless, some $60 \%$ remains unaccounted for in these analyses. With the inclusion of the ecological level of trust variable, a total of $52 \%$ for self-rated health and about $70 \%$ for depression were accounted for of the second level variance. The level of participation in organizational activity explained some of this variance for selfrated health, but was almost negligible in relation to depression.

Regarding our second research question, we found that the level of trust in an individual's neighbourhood was clearly associated with the operationalized health outcomes. Individuals living in wards possessing a low level of trust experienced a $50 \%$ increased risk of depression compared to individuals in high trust wards. For self-rated health, the social capital effect was 
Table 4. Multilevel logistic regression of depression, men and women 30-67 years HUNT II. 42,571 individuals nested within 155 wards. Odds ratios $(95 \% \mathrm{CI})$.

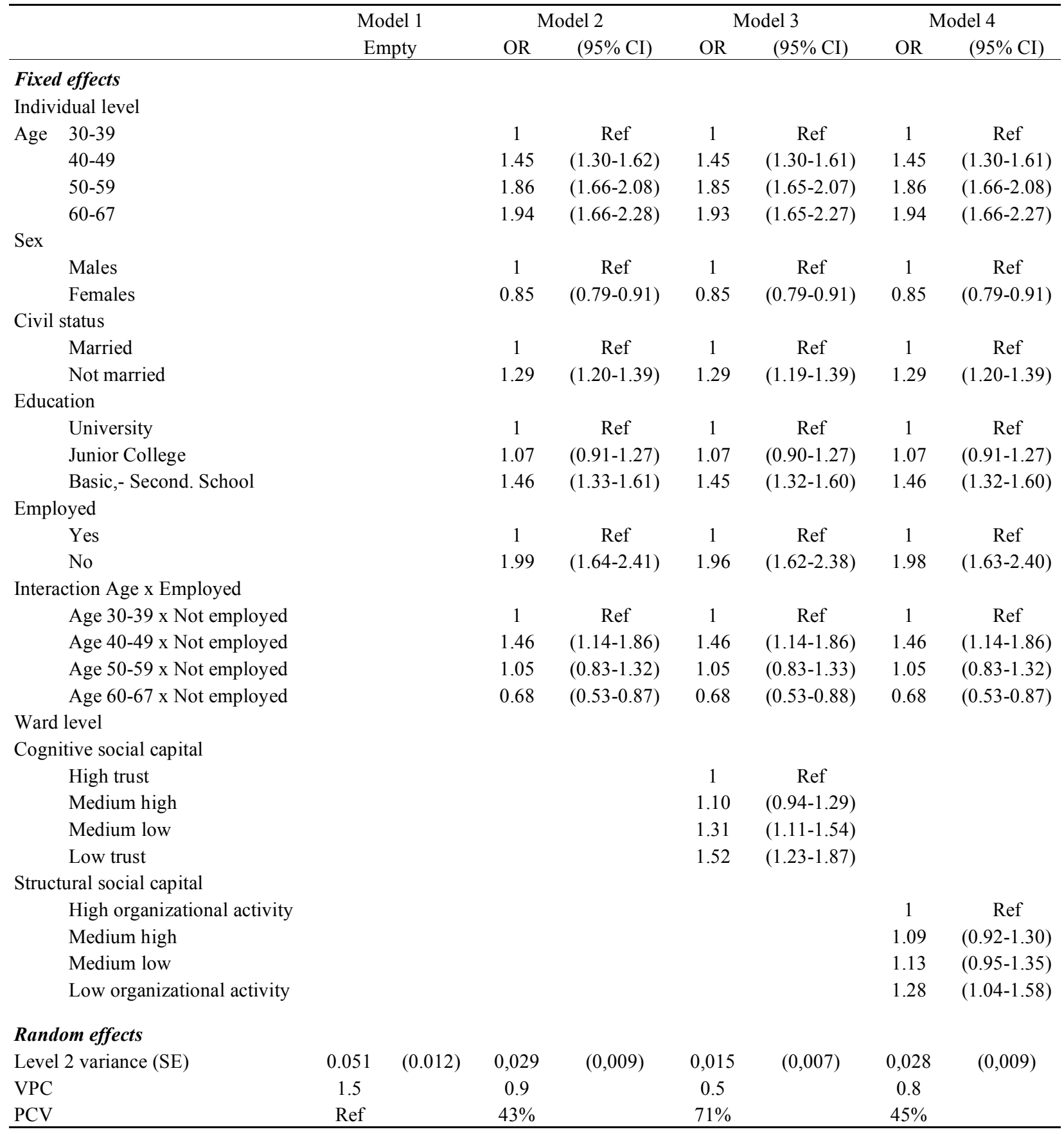

slightly weaker, with a $36 \%$ risk increase. Besides explaining some of the second level variance, this ecological construct also had a direct cross-level effect on individuals' health. The other social capital measure, participation in organizational activities, was found to be weak and gave inconsistent results.

Our first research question concerns a rather classical issue, often termed the composition/context debate. The fruitfulness of this dichotomy is debatable, and as has been argued, 'the distinction between "composition" and "context" may be more apparent than real'
(53, p. 125). The discussion concerns the extent to which area effects have an independent effect on the health of individuals. A common argument to set aside area-effect studies is that the models are mis-specified and the apparent area effect emerges due to individual level factors having been omitted in the analysis. The corresponding counter argument is that none of the individual factors or compositional characteristics in an area have emerged randomly. People move from one place to another for a variety of reasons, and they settle for an equal number of reasons (or limitations). 
Some of these individual characteristics might just as well be regarded as mediating factors rather than risk factors or confounders. As some geographers have stated: 'Places form people as much as places are formed from peoples' $(54$, p. 8$)$. This study included individual level attributes in accordance with previous studies of area effects.

The second research question, relating to the first, concerns social capital as an alleged area effect. This has importance for geographical health inequalities as well as bearings on individuals' health. The relationship poses problems in terms of interpretation. Regarding causality, the direction of this association could, in principle, go either way due to the interrelatedness between the outcomes at the individual level and our social capital predictors at the ecological level. Low trust could be the outcome of, as well as a determinant for, individual health (55). This poses a limitation especially for the association with depression - the varying degrees of trust can be a reflection of different prevalence's of depressed individuals in the wards. It is also questionable whether social capital is adequately operationalized and whether it is strictly spatially bounded.

Our findings contradict the conclusions from a review article on the social capital and health relationship in egalitarian societies (56): first, that social capital was less salient for individuals' health in egalitarian societies, and second, studies supporting this relationship applied a high geographical level, often using US states. Our study used the lowest available geographical level to measure social capital. The rationale behind this was that we would get more homogenous units intended to measure bonding social capital, i.e. the type of social capital between individuals with similar socio-economic status.

The social capital effect on individuals' health is a cross-level effect and refers to the main (or direct) effects of higher level units on outcomes at a lower level. This effect can, in principle, be confounded in the same way as individual level analyses can be confounded. We cannot rule out the possibility that other features of the neighbourhoods may be of importance, and as Diez Roux has suggested, we can make a conceptual distinction between two types of area level variables (57). On the one hand, we have the so-called 'integral' area features (no analogues at the individual level), such as the availability of health and social care, population density, certain types of regulations, etc. On the other hand, we have 'derived' features (summaries of individual properties), such as socioeconomic compositional characteristics, in/out migration, proportion single-person households, age/sex composition, etc. Related to the latter, the aggregation of individual level variables to represent an overindividual phenomenon can cause ambiguity of what this variable is actually measuring. A particular strength of this study is that the respondents were asked to relate their answer about trust to their imme- diate neighbourhood. This was not the case with the participation in organized activities, and may in fact explain our findings for this particular indicator. Furthermore, we would also have preferred adjustments for individual income, which is not available. It is not unlikely that some of the area variability and social capital effects could have been influenced. Ideally, we would have preferred to follow recent recommendations of using multiple measures of socio-economic position.

The underlying theme in this study concerns socioeconomic disparities in health that we observe at the individual level, or more correctly between aggregated positions in terms of stratification in society. The relation between social capital in individuals' neighbourhoods and social health inequalities may at first glance seem vague. Two apparently different perspectives may illustrate how features of the residential area may influence social health inequalities. The first is termed the 'neo-material', whilst the second is often termed the psychosocial. Briefly, the psychosocial explanation emphasizes the deteriorating health effects of relative social disadvantages and the accompanying feelings. The neo-materialists claim that the structural and material causes of inequalities are the main explanatory factors and not just perceptions of inequality and relative disadvantage. Applied to our study, the social capital and health relationship is considered a psychosocial interpretation, and the socio-economic inequalities in health in the wider society are partly caused by differing levels of social capital. These differing levels of social capital are expressed in extended social distance, differing levels of cohesion and solidarity, and is more stressful. The protective value of social capital for health thus varies according to neighbourhood. The neo-material interpretation, less occupied with perceptions of disadvantages, argues that these alleged area effects of social capital emerge because of differing levels of social investments in the neighbourhoods (e.g. in schools, health care). Our response to this discussion is that the latter interpretation, although highly relevant in other societies, has less value in the Norwegian welfare state. Variations in communities' provision of welfare services according to need are limited, and accordingly, local spending on welfare institutions is higher in disadvantaged communities. This redistribution effect makes the social capital and psychosocial interpretation more likely. Studies performed in Scandinavian welfare states may add pertinent knowledge to the debate of the relative magnitude of psychosocial versus neo-material factors in explaining socioeconomic disparities.

Similar to other studies applying a cross-sectional design, a cross-sectional multilevel study not only introduces the possibility of reverse causation, it also implies a zero lag-time between the ecological exposure (social capital) and individuals' health $(58,59)$. A zero lag-time is usually impossible, although impaired if the ecological exposure is stable over time. In order 
to reduce this misclassification bias this study applied health outcomes that to a larger extent respond to current exposures than mortality or diseases. It is simply more intuitive to expect an association with psychological and subjective health measures.

Selection bias is another unavoidable feature in population health surveys. Nevertheless, a non-responder study suggested no significant selection according to health or demography (60). The slightly lower participation in the youngest age groups does not affect this study as only adults (30+ years) are included. Another crucial bias in cross-sectional studies is the complexity of migration. The proposed area effect on individual health may also be misclassified. The effect of migration on geographical inequalities can, in principle, go either way, but in terms of area effects on individual health this constitutes a serious limitation and calls for longitudinal studies.

\section{CONCLUSION AND POLICY IMPLICATIONS}

The study indicates that over-individual characteristics, the level of trust associated with social capital in neighbourhoods, have significant impacts on self-rated health and depression, independent of individual-level factors. The findings give conditional support to assumptions that local social capital and neighbourhood still act as an influential framework or arena of life and health issues for people. The effects or presence or absence of such forms of local social capital or 'ties' may seem 'weak' (61) and marginal at first glance. Each effect, considered isolated, seems almost negligible and apparently disappears when their 'genuine' health effects are scrutinized. However, the sum effects of the captured protective factors or harmful factors may function as a 'sprinkling system'.

The area effect linked to the local physical environ- ment and their design and arrangements will stimulate and substantiate a well-functioning social life. Meeting places, access to low threshold exercise activities and green areas are just a few examples. They may facilitate social capital formations which enhance the local level of thriving and health. At the same time, neighbourhood inequalities in the socio-material structure may foster health inequalities in the population.

Mounting evidence that neighbourhood qualities and forms of social capital matter for the population's health has public health implications. To the point formulated, 'if social conditions rather than individual behaviour cause the problem, then society must share some of the responsibility for solving it' $(62$, p. 340). In an era of increasing individualism, collective health promoting actions initiated or supported by public or non-public efforts should be revisited.

Despite moderate support for the protective value of social capital for the health of individuals in this study, we do not recommend some kind of 'social engineering' to enhance social capital in society unless the more fundamental causes (63) have been addressed first. Nevertheless, if social capital is understood as a 'societal-wide capacity for inclusiveness, human rights, social justice, and full political and economic participation' then public health should invest in social capital $(23$, p. 408).

\section{ACKNOWLEDGEMENTS}

The Nord-Trøndelag Health Study (The HUNT Study) is a collaboration between the HUNT Research Centre, Faculty of Medicine, Norwegian University of Science and Technology (NTNU), Verdal, the National Institute of Public Health, the National Health Screening Service of Norway, and Nord-Trøndelag County Council. We also thank the reviewer and guest editors for valuable comments.

\section{REFERANSER}

1. Kawachi I, Berkman LF. Neighborhoods and health. Oxford University Press: Oxford, 2003.

2. Pickett KE, Pearl M. Multilevel analyses of neighbourhood socioeconomic context and health outcomes: a critical review. J Epidemiol Community Health 2001; 55: 111-122.

3. Macintyre S, Ellaway A. Neighborhoods and health: An overview. In: Kawachi I, Berkman L (eds). Neighborhoods and health. Oxford University Press: Oxford, 2003.

4. Wilkinson RG, Marmot MG. Social determinants of health: the solid facts, 2nd edn. WHO Regional office for Europe: Copenhagen, 2003.

5. Kawachi I, Berkman LF. Social epidemiology. Oxford University Press: New York, 2000.

6. Szreter S, Woolcock M. Health by association? Social capital, social theory, and the political economy of public health. Int J Epidemiol 2004; 33: 650-667.

7. Diez Roux AV. The study of group-level factors in epidemiology: rethinking variables, study designs, and analytical approaches. Epidemiol Rev 2004; 26: 104-111.

8. Yen IH, Syme SL. The social environment and health: a discussion of the epidemiologic literature. Anпи Rev Public Health 1999; 20: 287-308.

9. Schwartz S, Susser E, Susser M. A future for epidemiology? Annu Rev Public Health 1999; 20: 15-33.

10. Rose G. Sick individuals and sick populations. Int J Epidemiol 1985; 14: 32-38.

11. Rose G. The strategy of preventive medicine. Oxford University Press: Oxford, 1992. 
12. Schwartz S, Carpenter KM. The right answer for the wrong question: consequences of type III error for public health research. Am J Public Health 1999; 89: 1175-1180.

13. Wilkinson RG. Unhealthy societies. The afflictions of inequality. Routledge: London, 1996.

14. Wilkinson RG. Mind the gap. Hierarchies, health and human evolution. Weidenfeld \& Nicolson: London, 2000 .

15. Wilkinson RG. The impact of inequality. Routledge: London, 2005.

16. Mitchell R, Bartley M, Shaw M. Combining the social and the spatial: improving the Geography of health inequalities. In: Boyle PJ (ed). The Geography of health inequalities in the developed world: views from Britain and North America. Aldershot: England, 2005.

17. Putnam RD, Leonardi R, Nanetti RY. Making democracy work civic traditions in modern Italy. Princeton University Press: Princeton, N.J., 1993.

18. Harpham T, Grant E, Thomas E. Measuring social capital within health surveys: key issues. Health Policy Plan 2002; 17: 106-111.

19. Kawachi I, Kennedy BP, Glass R. Social capital and self-rated health: a contextual analysis. Am J Public Health 1999; 89: 1187-1193.

20. Wilkinson RG. Inequality and the social environment: a reply to Lynch et al. J Epidemiol Community Health 2000; 54: 411-413.

21. Marmot M, Wilkinson RG. Psychosocial and material pathways in the relation between income and health: a response to Lynch et al. BMJ 2001; 322: 1233-1236.

22. Pearce N, Davey Smith G. Is social capital the key to inequalities in health? Am J Public Health 2003; 93: 122-129.

23. Lynch J, Due P, Muntaner C, Smith GD. Social capital - is it a good investment strategy for public health? $J$ Epidemiol Community Health 2000; 54: 404-408.

24. Muntaner C. Commentary: Social capital, social class, and the slow progress of psychosocial epidemiology. Int $J$ Epidemiol 2004; 33: 674-680; discussion 700-674.

25. Kawachi I, Subramanian SV, Almeida-Filho N. A glossary for health inequalities. J Epidemiol Community Health 2002; 56: 647-652.

26. Kumlin S, Rothstein B. Making and breaking social capital - The impact of welfare-state institutions. Comp Polit Stud 2005; 38: 339-365.

27. Johnston R, Propper C, Sarker R, Jones K, Bolster A, Burgess S. Neighbourhood social capital and neighbourhood effects. Environ Plann A 2005; 37: 1443-1459.

28. Helliwell JF, Putnam RD. The social context of well-being. Philos Trans R Soc Lond B Biol Sci 2004; 359: $1435-1446$.

29. Sampson RJ, Raudenbush SW, Earls F. Neighborhoods and violent crime: A multilevel study of collective efficacy. Science 1997; 277: 918-924.

30. Wilkinson RG, Kawachi I, Kennedy BP. Mortality, the social environment, crime and violence. Sociol Health Illness 1998; 20: 578-597.

31. Kennedy BP, Kawachi I, Prothrow-Stith D. Income distribution and mortality: cross sectional ecological study of the Robin Hood index in the United States. BMJ 1996; 312: 1004-1007.

32. Kawachi I, Kennedy BP, Lochner K, Prothrow-Stith D. Social capital, income inequality, and mortality. Am $J$ Public Health 1997; 87: 1491-1498.

33. Sundquist J, Johansson SE, Yang M, Sundquist K. Low linking social capital as a predictor of coronary heart disease in Sweden: a cohort study of 2.8 million people. Soc Sci Med 2006; 62: 954-963.

34. Kim D, Subramanian SV, Gortmaker SL, Kawachi I. US state- and county-level social capital in relation to obesity and physical inactivity: a multilevel, multivariable analysis. Soc Sci Med 2006; 63: 1045-1059.

35. Almedom AM. Social capital and mental health: an interdisciplinary review of primary evidence. Soc Sci Med 2005; 61: 943-964.

36. Whitley R, McKenzie K. Social capital and psychiatry: review of the literature. Harvard Rev Psychiatry 2005; 13: 71-84.

37. Kim D, Kawachi I. A multilevel analysis of key forms of community- and individual-level social capital as predictors of self-rated health in the United States. J Urban Health 2006; 83: 813-826.

38. Subramanian SV, Kim DJ, Kawachi I. Social trust and self-rated health in US communities: a multilevel analysis. J Urban Health 2002; 79: S21-34.

39. Sundquist K, Yang M. Linking social capital and self-rated health: A multilevel analysis of 11,175 men and women in Sweden. Health Place 2007; 13: 324-334.

40. Veenstra G. Location, location, location: contextual and compositional health effects of social capital in British Columbia, Canada. Soc Sci Med 2005; 60: 2059-2071.

41. Veenstra G, Luginaah I, Wakefield S, Birch S, Eyles J, Elliott S. Who you know, where you live: social capital, neighbourhood and health. Soc Sci Med 2005; 60: 2799-2818. 
42. Ayuso-Mateos JL, Vazquez-Barquero JL, Dowrick C, Lehtinen V, Dalgard OS, Casey P, et al. Depressive disorders in Europe: prevalence figures from the ODIN study. Br J Psychiatry 2001; 179: 308-316.

43. Gogstad A, Bjerkedal T. More and more young people on disability pensions. Tidsskr Nor Laegeforen 2001; 121: $1452-1456$.

44. Mykletun A, Overland S, Dahl AA, Krokstad S, Bjerkeset O, Glozier N, et al. A population-based cohort study of the effect of common mental disorders on disability pension awards. Am J Psychiatry 2006; 163: 1412-1418.

45. Kaplan G, Baron-Epel O. What lies behind the subjective evaluation of health status? Soc Sci Med 2003; 56: 1669-1676.

46. Idler EL, Benyamini Y. Self-rated health and mortality: a review of twenty-seven community studies. $J$ Health Soc Behav 1997; 38: 21-37.

47. Fylkesnes K. Factors affecting self-evaluated general health status and the use of professional health care services. Institute of Community Medicine: Tromsø, 1991.

48. Schou MB, Krokstad S, Westin S. How is self-rated health associated with mortality? Tidsskr Nor Lageforen 2006; 126: 2644-2647.

49. Bjelland I, Dahl AA, Haug TT, Neckelmann D. The validity of the Hospital Anxiety and Depression Scale. An updated literature review. J Psychosom Res 2002; 52: 69-77.

50. Rasbash J, Steele F, Browne W, Prosser B. A User's Guide to MLwiN. Version 2.0. Centre for Multilevel Modelling. University of Bristol: Bristol, 2004.

51. Snijders TAB, Bosker RJ. Multilevel analysis. An introduction to basic and advanced multilevel modeling. Sage: London, 1999.

52. Merlo J, Yang M, Chaix B, Lynch J, Rastam L. A brief conceptual tutorial on multilevel analysis in social epidemiology: investigating contextual phenomena in different groups of people. J Epidemiol Community Health 2005; 59: 729-736.

53. Macintyre S, Ellaway A, Cummins S. Place effects on health: how can we conceptualise, operationalise and measure them? Soc Sci Med 2002; 55: 125-139.

54. Tunstall HV, Shaw M, Dorling D. Places and health. J Epidemiol Community Health 2004; 58: 6-10.

55. Portes A. Social capital: Its origins and applications in modern sociology. Annu Rev Sociol 1998; $24:$ 1-24.

56. Islam MK, Merlo J, Kawachi I, Lindstrom M, Gerdtham UG. Social capital and health: Does egalitarianism matter? A literature review. Int J Equity Health 2006; 5: 3.

57. Diez-Roux AV. Bringing context back into epidemiology: variables and fallacies in multilevel analysis. $A m J$ Public Health 1998; 88: 216-222.

58. Blakely TA, Kennedy BP, Glass R, Kawachi I. What is the lag time between income inequality and health status? J Epidemiol Community Health 2000; 54: 318-319.

59. Blakely TA, Woodward AJ. Ecological effects in multi-level studies. J Epidemiol Community Health 2000; 54: 367-374.

60. Holmen J, Midthjell K, Krüger Ø, Langhammer A, Lingaas Holmen T, Bratberg G, et al. The Nord-Trøndelag Health Study 1995-97 (HUNT 2). Objectives, contents, methods and participation. Nor J Epidemiol 2003; 13: 19-32.

61. Granovetter M. The strength of weak ties. Am J Sociol 1973; 78: 1360-1380.

62. Heymann J, Fischer A. Neighbourhoods, health research, and its relevance to public health policy. In: Kawachi I, Berkman LF (eds). Neighborhoods and health. Oxford University Press: Oxford, 2003.

63. Link BG, Phelan J. Social conditions as fundamental causes of disease. J Health Soc Behav 1995; Spec No: 80-94. 